\title{
Cerebrospinal Fluid Levels of $\alpha$-tocopherol and Neopterin in Patients with Dementia
}

\author{
Wolfyang Sattler ${ }^{1}$, Friedrich Leblhuber ${ }^{2}$, Johannes Walli ${ }^{2}$, Bernhard Widner ${ }^{3}$ and Dietmar Fuchs ${ }^{3, *}$ \\ ${ }^{1}$ Institute of Medical Biochemistry, University of Graz, A-8036 Graz, Austria \\ ${ }^{2}$ Department of Gerontology, Geriatric Clinic Wagner-Jauregg, A-4021 Linz, Austria \\ ${ }^{3}$ Institute of Medical Chemistry and Biochemistry, University of Innsbruck, A-6020 Innsbruck, Austria
}

(Received December 20, 1999)

\section{Summary}

In patients with different forms of dementia we compared serum and cerebrospinal fluid concentrations of $\alpha$-tocopherol (vitamin-E), the major circulating lipid-soluble antioxidant, and neopterin, a product released from monocytic cells upon stimulation with interferon- $\gamma$. A significant inverse correlation was found between the concentrations of both compounds in the cerebrospinal fluid. The data point to a role of oxidative stress to reduce antioxidant levels in elderly demented patients with neurodegenerative diseases.

Key words : $\alpha$-tocopherol, Neopterin, Dementia, Oxidative stress, Alzheimer type dementia

\section{Introduction}

The etiology of neuronal death in neurodegenerative diseases remains unclear (1), most of which are characterized by a gradually progressing course. There is data indicating that excitotoxicity and oxidative damage could be involved in the pathogenesis of these diseases $(2,3)$. Localized immune reaction and production of pro-inflammatory cytokines has been documented in the brain of patients with various neurodegenerative disorders, e.g., the synthesis of cytokines such as interleukin-6 was demonstrated immunohistochemically in plaques of Alzheimer's disease brains and confirms a cytokine-mediated process as part of the pathology (4). However, altered immune cell responsiveness and abnormal concentrations of immune mediators were not only described in neurodegenerative diseases but also in the healthy

§Corresponding author: Fritz Pregl Strasse 3, A-6020 Innsbruck, Austria. Tel. +43512 507 3519; fax: +43 512 507 2865; E-mail address: dietmar.fuchs@uibk.ac.at elderly $(2,5)$.

Activated phagocytic cells produce a variety of reactive oxygen species, and thus are an important source of oxidative stress during diseases. However, methods applicable for the assessment of oxidative stress in vivo are limited and suffer from low sensitivity. A strong association exists between the capacity of monocytes/macrophages to release reactive oxygen species and the amount of neopterin upon stimulation with interferon- $\gamma(6,7)$. Moreover, neopterin derivatives were described recently to be capable of enhancing effects of reactive oxygen species (8). Thus, increased neopterin concentrations in body fluids of patients suffering from immunological disorders not only reflect immune activation but also can be regarded as an indicator of oxidative stress induced by reactive oxygen species released during the cellular immune response. Since neopterin is a stable product, the monitoring of neopterin concentrations is easily performed. As in patients with, e.g., virus infections, autoimmune diseases or cancer, increased neopterin concentrations were also found 
in the serum of patients with Alzheimers disease and with Huntingtons disease, and an inverse correlation existed between Mini-Mental-State and neopterin concentrations $(9,10)$. However, only a slight increase of neopterin concentrations has been found in the CSF of these patients, and importantly, neopterin concentrations in CSF usually were lower than in paired serum or plasma specimens. This data imply that the increased concentrations of neopterin in the serum are not a result of overproduction of the compound inside the brain.

Lipid peroxidation by radical reactions is considered to contribute to tissue damage in neurodegenerative disorders. $\alpha$-Tocopherol (vitamin-E) is a major lipid antioxidant which is capable of terminating free radical chain reactions. During this reaction $\alpha$ tocopherol is oxidized, and concentrations of $\alpha$-tocopherol were found decreased in the cerebrospinal fluid (CSF) and serum of patients with, e.g., Alzheimers disease (11-13). Vice versa, treatment with seleginine and $\alpha$-tocopherol was shown to slow the clinical deterioration of the disease $(14,15)$. Because $\alpha$-tocopherol is a lipid-soluble vitamin that interacts with cell membranes, traps free radicals, and interrupts the chain reaction that damages cells, deficiency of $\alpha$-tocopherol in the brain might be a consequence of increased oxidative stress, and the efficacy of $\alpha$-tocopherol in the treatment of patients could be due to its antioxidative capacity: It is also noteworthy that patients suffering from familial inherited vitamin $\mathrm{E}$ (FIVE) deficiency develop almost identical neurological svndromes as observed in patients suffering from Friedreichs ataxia.

\section{Patients and Methods}

In this study we compared serum and CSF concentrations of $\alpha$-tocopherol and neopterin in 10 patients with different forms of dementia (age: 70.8 \pm 13.8 years; mean \pm S.D.) -5 patients with Alzheimers disease according to DSM-IIIR criteria [16] (aged 72-85 years), 3 patients with vascular dementia (aged 77, 81, 85 years), and 2 patients with Huntingtons disease (aged 35 and 65 years)-and in 3 controls $(57,70,77=68.0 \pm 10.2$ years $)$. CSF and serum concentrations of $\alpha$-tocopherol were measured by reversed phase high pressure liquid chromatography (HPLC) with fluorescence detection (290 nm excitation and $325 \mathrm{~nm}$ emission wavelength), and neopterin concentrations in serum and CSF were determined by ELISA (BRAHMS; Berlin, Germany). In addition urinary neopterin concentrations ( $\mu \mathrm{mol} / \mathrm{mol}$ creatinine) were measured by HPLC in early morning spot urine specimens (6).
Data were analyzed by non-parametric Mann Whitney U-test, correlation analyses were done by Spearman rank statistics. P-values below 0.05 were considered to indicate statistical significance.

\section{Results}

Serum concentrations of $\alpha$-tocopherol were 23.7 $\pm 3.88 \mu \mathrm{M}$ (mean \pm S.D.) and at the lower side compared to the 3 controls $(27.2 \pm 4.59 \mu \mathrm{M}$; n.s.; Fig. $1)$, whereas serum neopterin concentrations $(9.46$ $\pm 2.97 \mathrm{nM})$ were higher than in controls (6.83 \pm $2.39 \mathrm{nM} ; \mathrm{p}=0.066)$. CSF $\alpha$-tocopherol concentrations generally were 400 -fold lower than serum levels, and again they were slightly lower in patients $(0.056 \pm 0.017 \mu \mathrm{M})$ than in controls $(0.064 \pm 0.022$ $\mu \mathrm{M}$; n.s.). Neopterin concentrations in CSF were approximately half of the concentrations found in serum, and levels in patients with dementia (5.98 \pm
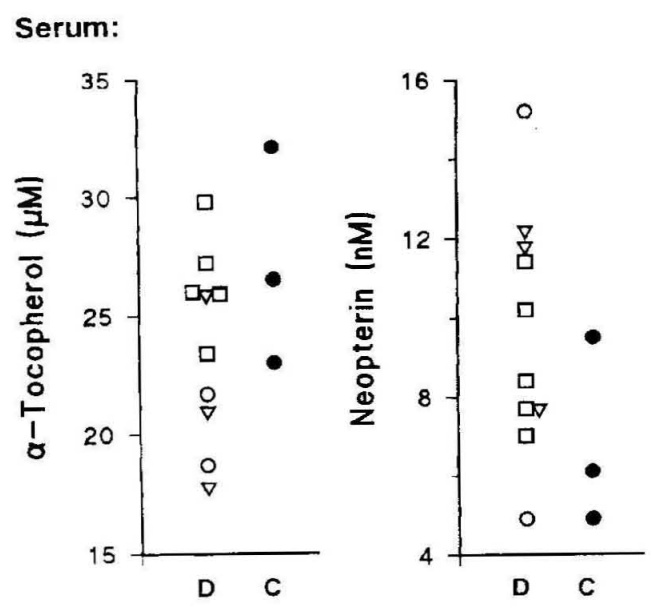

Cerebrospinal fluid:
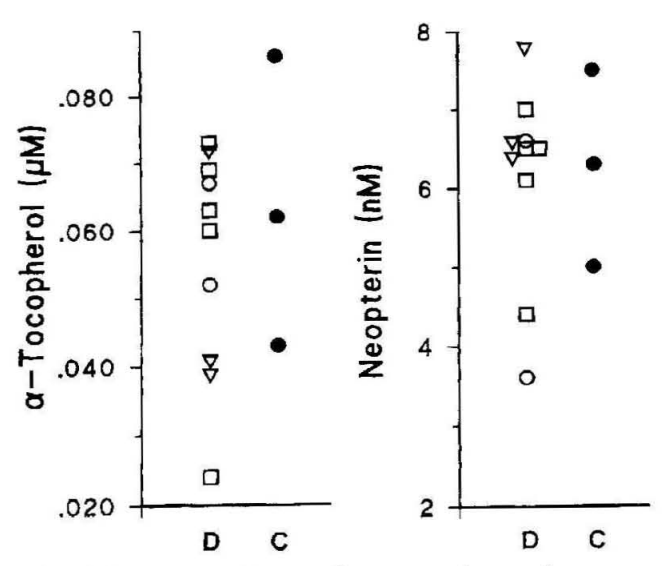

Figure 1. Concentrations of neopterin and $\alpha$-tocopherol in serum and cerebrospinal fluid of patients with dementias (D): 5 with Alzheimers disease (open squares), 2 with Huntingtons disease (open circles), 3 with vascular dementia (open triangles) and controls $(\mathbf{C}): n=3$ (closed circles). 


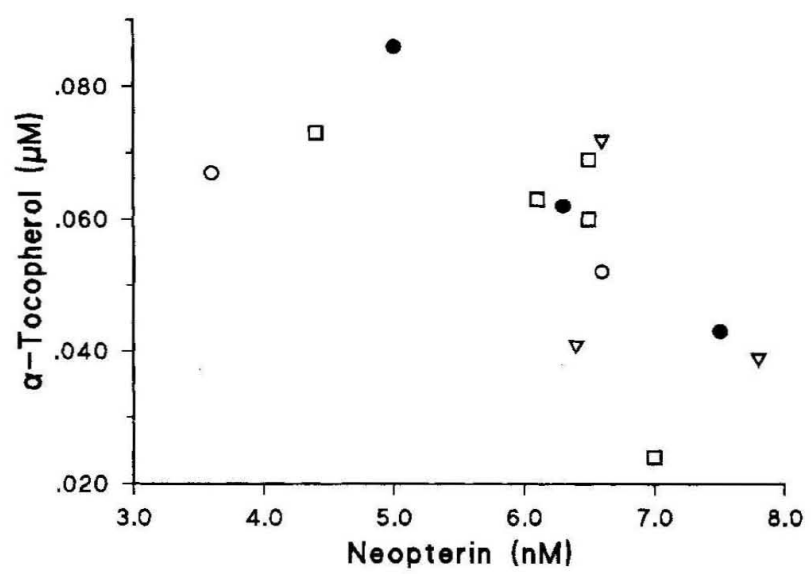

Figure 2. Cerebrospinal fluid concentrations of $\alpha$-tocopherol and neopterin in 10 patients with dementias: 5 with Alzheimers disease (open squares), 2 with Huntingtons disease (open circles), 3 with vascular dementia (open triangles) and 3 controls (closed circles) (all data, $n=13$ : $\mathrm{rs}=-0.678, \mathrm{p}<0.01$; dementias only, $\mathrm{n}=9$ : $\mathrm{rs}=-0.579, \mathrm{p}$ $<0.05)$.

$1.30 \mathrm{nM})$ totally overlapped with controls $(6.27 \pm$ 1.25 ; n.s.). Actually all of the patients with dementia had lower neopterin concentrations in CSF than in serum ( $\mathrm{U}=2.95, \mathrm{p}=0.003$; paired rank test $)$. Patients had elevated urinary neopterin concentrations (mean \pm S.D.: $235 \pm 87 \mu \mathrm{mol} / \mathrm{mol}$ creatinine), and all but one patients had higher neopterin concentrations than the 3 controls (range: 132-177 $\mu \mathrm{mol} / \mathrm{mol}$ creatinine). The one patient with Huntingtons disease was 35 years old and had a urinary neopterin concentration of $133 \mu \mathrm{mol} / \mathrm{mol}$ creatinine.

There existed an inverse correlation between CSF neopterin and $\alpha$-tocopherol concentrations when specimens of all individuals were compared as well as when only patients with dementia were considered (Fig. 2). In addition, $\alpha$-tocopherol concentrations in CSF were significantly associated with their corresponding serum levels $(r s=0.599, \mathrm{p}<0.05)$ and also urinary neopterin concentrations correlated with serum concentrations ( $\mathrm{rs}=0.652, \mathrm{p}<0.05$ ). There was no correlation between age and neopterin or $\alpha$ tocopherol concentrations, and there were no other significant relationships between the parameters investigated.

\section{Discussion}

Our data reveal no drastic increase of neopterin concentrations in patients with dementia compared to controls of similar age. However, the low number of controls does not allow a firm conclusion, but is obvious that with increasing age neopterin concentrations in healthy individuals are increasing (17).
This is true for serum and CSF, and it is consistent with the view that neopterin concentrations as other indicators of immune system activation are increasing in body fluids with increasing age $(2,15)$. From neopterin concentrations it appears that the relative increase with age is more pronounced in CSF than in serum (17). Neopterin concentrations found in CSF of our patients not only fit well to the levels in healthy controls in this study but also to data available in the literature (17). This result agrees well with recent observations on low or undetectable concentrations of neopterin, interferon- $\gamma$ and tumor necrosis factor- $\alpha$ in patients with Alzheimers disease (18). In our study, we found a significant inverse correlation between neopterin and $\alpha$-tocopherol concentrations in the CSF of patients suffering from different forms of dementia. Neopterin indicates immune activation and is indirectly and probably directly associated with oxidative stress. Thus, the inverse correlation found between neopterin and $\alpha$ tocopherol in CSF is in line with the hypothesis that oxidative stress may be involved in the decrease of the antioxidant $\alpha$-tocopherol and this could be part of the pathogenesis of these neurodegenerative disorders which cause dementia.

A positive correlation existed between $\alpha$-tocopherol concentrations in serum and CSF. As serum concentrations of $\alpha$-tocopherol are approximately 400fold higher than that in CSF, it appears plausible that the decreased $\alpha$-tocopherol concentrations in CSF might be a reflection of decreased $\alpha$-tocopherol concentrations in the peripheral blood rather than oxidation of $\alpha$-tocopherol within the blood brain barrier. The decreased availability of $\alpha$-tocopherol in the CSF of older aged people may represent a cofactor for the development of dementia. This scenario is also in line with the fact that treatment with reductants like $\alpha$-tocopherol or seleginine is of benefit for patients with neurodegenerative diseases $(14,15)$. It is interesting to note that increased neopterin and decreased $\alpha$-tocopherol levels were observed in all three groups of patients with dementia. Increased serum neopterin concentrations have also been described earlier in a patient with classic Creutzfeld-Jacob disease, again with lower concentrations in the CSF than in serum (19), and the same situation appears to exist in patients with Parkinsons disease (20). Yet, increased serum neopterin is not a general finding in neurodegenerative disorders, rather it appears related to older age and to the development of dementia because patients with amyotrophic lateral sclerosis, who are usually much younger than patients with, e.g., Alzheimers disease, usually present with neopterin concentrations in 
serum and CSF within the normal range of healthy controls (2I). Also in our 35-years old patient with Huntingtons disease neopterin as well as $\alpha$-tocopherol concentrations in the CSF are well within the normal range of the corresponding age group. Decreased concentrations of $\alpha$-tocopherol have been described in serum of patients with Alzheimers disease already before (11-13), but low $\alpha$-tocopherol concentrations together with increased neopterin concentrations were also found in nonagenarians without dementia (22).

Immune activation seems to increase in individuals with older age, irrespective of the development of neurodegenerative disorders. In the same way, oxidative stress increases and contributes to a lower antioxidant capacity and lower $\alpha$-tocopherol with increasing age which will support the development of neurodegenerative diseases in susceptible individuals. From our data it still remains unclear whether the development of neurodegenerative disorders involves oxidative stress greater than normal, which may further accelerate the loss of antioxidants like $\alpha$ tocopherol.

Although correlation does not necessarily indicate a cause-effect relationship, the finding of lowest $\alpha$ tocopherol in CSF of patients with highest neopterin concentrations indicates that chronic immune activation and oxidative stress seem to contribute to depletion of $\alpha$-tocopherol, and decreased concentrations of the antioxidant in patients are unlikely to be just a result of reduced dietary intake.

\section{Acknowledgement}

This work was supported by the Austrian Ministry of Science and Transport.

\section{References}

1. Geldmacher DS and Whitehouse PJ, Evaluation of dementias, New Engl. J. Med. 335: 30-36, 1996.

2. Beal MF Aging, energy, and oxidative stress in neurodegenerative disease, Ann. Neurol. 38: 357-366, 1995.

3. Jenner $P$, Oxidative damage in neurodegenerative disease, Lancet 344: 796-798, 1994.

4. Frei K, Malipicro UV, Leist TP, Zinkernagel RM, Schwab ME and Fontana A, On the cellular source and function of interleukin- 6 produced in the central nervous svstem, Eur. J. Immunol. 19: 689-694, 1989.

5. Mooradian AD, Reed RL and Scuderi P, Serum levels of tumor necrosis factor alpha, interleukin-l alpha and beta in healthy elderly subjects, Age 14: 61-64, 1991.

6. Fuchs D, Weiss, $G$ and Wachter H, Neopterin, biochemistry and clinical use as a marker for cellular immune reactions, Int. Arch. Allergy Immunol. 101: 1-6, 1993.
7. Widner B, Baier-Bitterlich G, Wede I, Wirleitner B, Wachter $\mathrm{H}$ and Fuchs D, Neopterin: Indicator of oxidative stress and part of the cytotoxic armature of activated macrophages in humans. Pteridines 9: 91-102, 1998.

8. Murr C, Baier-Bitterlich G, Fuchs D, Werner ER, Esterbauer $\mathrm{H}$, Pfleiderer $\mathrm{W}$ and Wachter $\mathrm{H}$, Effects of neopterin-derivatives on $\mathrm{H}_{2} \mathrm{O}_{2}$-induced luminol chemiluminescence: mechanistic aspects, Free Radical Biol. Med. 21: 449-56, 1996.

9. Leblhuber F, Walli J, Jellinger K, Tilz GP, Widner B, Laccone F and Fuchs D, Activated immune system in patients with Huntington's disease, Clin. Chem. Lab. Med. 36: 747-750, 1998.

10. Leblhuber F, Walli J, Demel U, Tilz GP, Widner B and Fuchs D, Increased serum neopterin concentrations in patients with Alzhcimer's disease, Clin. Chem. I ab. Med. 37: 429-431, 1999.

11. Jeandel C, Nicolas MB, Dubois F, Nabet-Belleville F, Penin $F$ and Cuny $G$, Lipid peroxidation and free radical scavengers in Alzheimers disease, 35: 275-282, 1989.

12. Toghi H, Abe T, Nakanishi M, Hamato F, Sasaki K and Takahashi $S$, Concentrations of $\alpha$-tocopherol and its quinone derivative in cerebrospinal fluid from patients with vascular dementia of the Binswanger $\pi p c$ and Alzheimer type dementia, Neurosci. Lett. 174: 73-76. 1994.

13. Zaman Z, Roche S, Fielden P, Frost PG, Niriclla DC and Carles ACD, Plasma concentrations of vitamins A and $\mathrm{E}$ and carotenoids in Alzheimers disease, Age Ageing, 21: 91-94, 1992.

14. Sano M, Ernesto C, Thomas, RG, Klauber MR, Schater K, Grundman M, Woodbury P, Growdon J, Cotman CW, Pfeiffer, E, Schneider, LS and Thal, LJ, for the members of the Alzhcimers disease cooperative studs: A controlled trial of seleginine, alpha-tocopherol, or both as treatment for Alzhcimers disease, New Engl. J. Med. 336: 1216-1222, 1997.

15. Small GW, Treatment of Alzheimers disease: current approaches and promising developments, Am. J. Med. 104: 32s-38s, 1998.

16. McKhann G, Drachmann D, Folstein M, Katzmann R, Price D and Stadlan EM, Clinical Diagnosis of Alzheimer's disease Report of the NINCDS-ADRDA Work Group under the auspices of the Department of Health and Human Services Task Force of Alzheimcr's disease, Neurology 34: 934-944, 1984.

17. Hagberg L, Dotevall L, Norkrans G, Larsson M, Wachter $\mathrm{H}$ and Fuchs D, Cerebrospinal fluid neopterin concentrations in central nervous system infection. J. Infect. Dis. 168: 1285-1288, 1993.

18. Engelborghs S, De Brabander M, De Cree J, DHooge $R$, Geerts $H$, Verhaegen $H$ and De Deyn $P P$, Unchanged levels of interleukins, neoptcrin, interferongamma and tumor necrosis factor-alpha in cerebrospinal fluid of patients with dementia of the Alzheimer type, Neurochem. Int. 34: 523-530, 1999.

19. Leblhuber F, Walli J, Tilz GP, Jellinger K, Wachter $H$ and Fuchs D, Increased serum neopterin concentrations in a patient with Creutzfeldt-Jakob diseasc. J. Neurol. Neurosurg. Psych. 61: 211-212, 1996. 
20. Widner B, Leblhuber F, Fuchs D, Increased neopterin concentrations in patients with Parkinsons disease. J. Neurol Neurosur Psychiatry, submitted for publication.

21. Westarp ME, Fuchs D, Bartmann P, Hoff-Jörgensen R, Clausen J, Wachter $\mathrm{H}$ and Kornhuber $\mathrm{HH}$, Amyotrophic lateral sclerosis-an enigmatic disease with B-cel- lular and anti-retroviral immune responses, Eur. J. Med. 2: 327-332, 1993.

22. Solichova D, Melichar B, Svobodova I, Blaha V, Zadak $Z$., Fluorescence analysis of antioxidant vitamins and neopterin in nonagenarians, Biomed Chromat 13: $117-$ $118,1999$. 